\title{
Reliability for Lindley Distribution with an Outlier
}

\author{
Hossein Jabbari Khamnei
}

Department of Statistics, Faculty of mathematical sciences, University of Tabriz, Tabriz, Iran

Keywords.Lindley Distribution, Maximum Likelihood Estimator, Newton-Raphson Method, Outlier.

Abstract. In this paper, we consider the problem of estimating $R=P(Y<X)$, when $Y$ has lindley distribution with parameter $a$ and $x$ has lindley distribution with presence of one outlier with parameters $b$ and $c$, such that $X$ and $Y$ are independent. The maximum likelihood estimator of $R$ is derived and some results of simulation studies are presented.

\section{Introduction}

In reliability context inferences about $R=P(Y<X)$, when $\mathrm{X}$ and $\mathrm{Y}$ are independently distributed, are a subject of interest.For example in mechanical reliability of a system if $X$ is the strength of a component which is subject to stress $\mathrm{Y}$, then $\mathrm{R}$ is a measure of system performance. The system fails, if at any time the applied stress is greater than its strength. Stress-strength reliability has been discussed in Kapur and Lamberson (1977). Sathe and Dixit (2001) have done estimation of R in the negative binomial distribution. Baklizi and Dayyeh (2003) have done shrinkage estimation of $\mathrm{R}$ in exponential case, and recently Deiri (2011) has done estimation of R with presence of two outliers in the exponential and gamma cases, respectively.Jafari (2011) has obtained the moment, maximum likelihood and mixture estimators of $\mathrm{R}$ in Rayleigh distribution in the presence of one outlier and Jabbari, Abolhasani and Fathipour (2012) have discussed the estimation of $\mathrm{R}$ in the six parameter generalized Burr XII distribution with transformation method.

In this paper, we obtain the maximum likelihood estimator of $\mathrm{R}$ for lindley distribution with presence of one outlier generated from the same distribution.

The probability density function of the lindley distribution with parameter of $a$ is given by:

$f(y ; a)=\frac{a^{2}}{1+a}(1+y) e^{-a y}, x>0, a>0$.

In this paper we assume that the random variables $\left(Y_{1}, Y_{2}, \ldots, Y_{m}\right)$ have lindley distribution with parameter $a$ and the random variables $\left(X_{1}, X_{2}, \ldots, X_{n}\right)$ are such that one of them is from lindley distribution with parameter $c$ and the remaining (n-1) random variables are from lindley distribution with parameter $b$

The paper is organized as follows:

In section 2, we obtain the joint distribution of $\left(X_{1}, X_{2}, \ldots, X_{n}\right)$ in the presence of one outlier. Section 3 and section 4 discusses the method of maximum likelihood estimators of parameters and the MLE of $R$ respectively. In section 5 simulation studies are presented and the results are summarized in section 6 .

\section{Joint distribution of $X 1, X 2, \ldots, X n$ in presence of an outlier}

Assume $\left(X_{1}, X_{2}, \ldots, X_{n}\right)$ are such that one of them is distributed with p.d.f $g(x, c)$ as lindley $(c)$ and remaining $(n-1)$ of them are distributed with p.d.f $f(x, b)$ as lindley $(b)$. The joint distribution of $\left(X_{1}, X_{2}, \ldots, X_{n}\right)$ can be expressed as

$$
f\left(x_{1}, x_{2}, \ldots, x_{n} ; b, c\right)=\frac{(n-1) !}{n !} \prod_{i=1}^{n} f(x, b) \sum_{i=1}^{n} \frac{g\left(x_{i} ; c\right)}{f\left(x_{i} b\right)}
$$




$$
\begin{aligned}
& =\frac{(n-1) !}{n !} \frac{b^{2 n}}{(1+b)^{n}} \prod_{i=1}^{n}\left(1+x_{i}\right) e^{-b \sum_{i=1}^{n} x_{i}} \sum_{i=1}^{n} \frac{\frac{c^{2}}{1+c}\left(1+x_{i}\right) e^{-c x_{i}}}{\frac{b^{2}}{1+b}\left(1+x_{i}\right) e^{-b x_{i}}} \\
& =\frac{(n-1) !}{n !} \frac{b^{2 n-2}}{(1+b)^{n-1}} \frac{c^{2}}{1+c} \prod_{i=1}^{n}\left(1+x_{i}\right) e^{-b \sum_{i=1}^{n} x_{1}} \sum_{i=1}^{n}\left(1+x_{i}\right) e^{x_{i}(b-c)}
\end{aligned}
$$

See Dixit (1989), Dixit and Nasiri (2001), and Nasiri and Pazira (2009). From (1), the marginal distribution of $X$ is

$$
f(x ; b, c)=\frac{1}{n} \frac{c^{2}}{1+c}(1+x) e^{-c x}+\frac{n-1}{n} \frac{b^{2}}{1+b}(1+x) e^{-b x} ; x, b, c>0
$$

We will use (2) to obtain $R=P(Y<X)$

\section{Maximum likelihood estimators of parameters}

Let $\left(Y_{1}, Y_{2}, \ldots, Y_{m}\right)$ be a random sample for $Y$ with pdf,

the log likelihood function is given by

$$
f(y ; a)=\frac{a^{2}}{1+a}(1+y) e^{-a y}, x, a>0
$$

$$
L(a)=2 m \ln a-m \ln (1+a)+\sum_{i=1}^{m} \ln \left(1+y_{i}\right)-a \sum_{i=1}^{m} y_{i}
$$

Taking the derivative with respect to $a$ and equating to 0 , we obtain the MLE of $a$ as

$$
\hat{a}=\frac{m-\sum_{i=1}^{m} y_{i} \pm \sqrt{\left(\sum_{i=1}^{m} y_{i}-m\right)^{2}+8 m \sum_{i=1}^{m} y_{i}}}{2 \sum_{i=1}^{m} y_{i}}
$$

Now let $X 1, X 2, \ldots, X n$ be a random sample for $X$ with presence of one outlier with pdf,

$$
f(x ; b, c)=\frac{1}{n} \frac{c^{2}}{1+c}(1+x) e^{-c x}+\frac{n-1}{n} \frac{b^{2}}{1+b}(1+x) e^{-b x} ; x, b, c>0 .
$$

From (1), the log likelihood function is given by

$$
\begin{gathered}
L(b, c)=\ln \left(\frac{(n-1) !}{n !}\right)+(2 n-2) \ln d-(n-1) \ln (1+b)+2 \ln c-\ln (1+c) \\
+\sum_{i=1}^{n} \ln \left(1+x_{i}\right)-b \sum_{i=1}^{n} x_{i}+\ln \left(\sum_{i=1}^{n} e^{x_{i}(b-c)}\right.
\end{gathered}
$$

Taking the derivatives with respect to $b$ and $c$ and equating the results to 0 , we obtain the normal equations as

$$
\begin{gathered}
\frac{\partial L(b, c)}{\partial b}=\frac{2 n-2}{b}-\frac{n-1}{1+b}-\sum_{i=1}^{n} x_{i}+\frac{\sum_{i=1}^{n} x_{i} e^{x_{i}(b-c)}}{\sum_{i=1}^{n} e^{x_{i}(b-c)}} \\
\frac{\partial L(b, c)}{\partial c}=\frac{2}{c}-\frac{1}{1+c}-\frac{\sum_{i=1}^{n} x_{i} e^{x_{i}(b-c)}}{\sum_{i=1}^{n} e^{x_{i}(b-c)}}
\end{gathered}
$$

There is no closed-form solution to this system of equations, so we will solve for $\hat{b}$ and $\hat{c}$ iteratively, using the Newton-Raphson method. In our case we will estimate $\hat{\beta}=(\hat{b}, \hat{c})$ iteratively:

$$
\hat{\beta}_{i+1}=\hat{\beta}_{i}-G^{-1} g
$$

where $g$ is the vector of normal equations for which we want

With

$$
g=\left[g_{1} g_{2}\right]
$$

$g_{1}=\frac{2 n-2}{b}-\frac{n-1}{1+b}-\sum_{i=1}^{n} x_{i}+\frac{\sum_{i=1}^{n} x_{i} e^{x_{i}(b-c)}}{\sum_{i=1}^{n} e^{x_{i}(b-c)}}$

$g_{2}=\frac{2}{c}-\frac{1}{1+c}-\frac{\sum_{i=1}^{n} x_{i} e^{x_{i}(b-c)}}{\sum_{i=1}^{n} e^{x_{i}(b-c)}}$ 
and $G$ is the matrix of second derivatives

where

$$
G=\left[\begin{array}{ll}
\frac{d g_{1}}{d b} & \frac{d g_{1}}{d c} \\
\frac{d b_{2}}{d b} & \frac{d g_{2}}{d c}
\end{array}\right]
$$

$\frac{d g_{1}}{d b}=\frac{2-2 n}{b^{2}}+\frac{n-1}{(1+b)^{2}}+\frac{\sum_{i=1}^{n} x_{i}^{2} e^{x_{i}(b-c)}}{\sum_{i=1}^{n} e^{x_{i}(b-c)}}-\left(\frac{\sum_{i=1}^{n} x_{i} e^{x_{i}(b-c)}}{\sum_{i=1}^{n} e^{x_{i}(b-c)}}\right)^{2}$

$\frac{d g_{1}}{d b}=-\frac{\sum_{i=1}^{n} x_{i}^{2} e^{x_{i}(b-c)}}{\sum_{i=1}^{n} e^{x_{i}(b-c)}}+\left(\frac{\sum_{i=1}^{n} x_{i} e^{x_{i}(b-c)}}{\sum_{i=1}^{n} e^{x_{i}(b-c)}}\right)^{2}$

$\frac{d g_{1}}{d b}=\frac{-2}{c^{2}}+\frac{1}{(1+c)^{2}}+\frac{\sum_{i=1}^{n} x_{i}^{2} e^{x_{i}(b-c)}}{\sum_{i=1}^{n} e^{x_{i}(b-c)}}-\left(\frac{\sum_{i=1}^{n} x_{i} e^{x_{i}(b-c)}}{\sum_{i=1}^{n} e^{x_{i}(b-c)}}\right)^{2}$

The Newton-Raphson algorithm converges, as our estimate of $b$ and $c$ change by less than a tolerated amount with each successive iteration, to $\hat{b}$ and $\hat{c}$.

\section{The maximum likelihood estimator of $R$}

Let $Y \sim$ lindley $(a)$ with pdf $h(y ; a)$ and $X$ be distributed with pdf $f(x ; b, c)$ given in (2). The parameter $R$ we want to estimate is

$$
\begin{array}{rl}
R=P(Y<X)=\int_{0}^{\infty} \int_{0}^{x} & h(y ; a) f(x ; b, c) d y d x \\
& =\frac{1}{b} \int_{0}^{\infty} \int_{0}^{x} \frac{a^{2}}{1+a}(1+y) e^{-a y} \frac{c^{2}}{1+c}(1+x) e^{-c x} d y d x \\
+ & \frac{n-1}{n} \int_{0}^{\infty} \int_{0}^{x} \frac{a^{2}}{1+a}(1+y) e^{-a y} \frac{b^{2}}{1+b}(1+x) e^{-b x} d y d x \\
& =\frac{1}{n}\left[\frac{c^{2}\left(c(1+c)+(1+c)(3+c) a+(3+2 c) a^{2+} a^{3}\right.}{(1+c)(1+a)(c+a)^{3}}\right] \\
+ & \frac{n-1}{n}\left[\frac{b^{2}\left(b(1+b)+(1+b)(3+b) a+(3+2 b) a^{2}+a^{3}\right.}{(1+b)(1+a)(b+a)^{3}}\right]
\end{array}
$$

Thus, by invariant property for MLEs, the MLE of $R$ is

$$
\begin{aligned}
& \hat{R}=\frac{1}{2}\left[\frac{\hat{c}^{2}\left(\hat{c}(1+\hat{c})+(1+\hat{c})(3+\hat{c}) \hat{a}+(3+2 \hat{c}) \hat{a}^{2}+\hat{a}^{3}\right)}{(1+\hat{c})(1+\hat{a})(\hat{c}+\hat{a})^{3}}\right] \\
& +\frac{n-1}{n}\left[\frac{\hat{b}^{2}\left(\hat{b}(1+\hat{b})+(1+\hat{b})(3+\hat{b}) \hat{a}+(3+2 \hat{b}) \hat{a}^{2}+\hat{a}^{3}\right.}{(1+\hat{b})(1+\hat{a})(\hat{b}+\hat{a})}\right]
\end{aligned}
$$

where $\hat{a}, \hat{b}$, and $\hat{c}$ can be obtained from (3) and (6).

\section{Simulation Study}

In this section we generate random numbers from lindley distribution (with and without outlier) with accept-reject method by Maple software. Using these samples and the Newton-Raphson method we obtain the maximum likelihood estimators of parameters $a, b$ and $c$. Then we use them to calculate the MLE of $R$. The values of biases and MSEs of these estimates are presented in table1, for $a=1, b=2$ and $\mathrm{c}=1.6,1.7,1.8,1.9,2.1,2.2,2.3,2.4,2.5,3,4$ and in table 2, for $a=1, b=2$, and the same values of $c$. All the results are based on 100 replications.

\section{Conclusion}

Acording to the results of simulation, when the value of parameters $b$ and $c$ are close to each other, the biases and MSEs are often around zero and when the difference between $b$ and $c$ is greater than 1 , the biases and MSEs increase. 
Table1: Biases and (MSE)s of the MLEs of $R$, for $a=1, b=2$, and different values of $c$

\begin{tabular}{|c|c|c|c|c|c|c|c|c|c|}
\hline $\begin{array}{c}(\mathrm{m}, \mathrm{n}) \rightarrow \\
\mathrm{c} \downarrow\end{array}$ & $(30,30)$ & $(30,20)$ & $(30,10)$ & $(20,30)$ & $(20,20)$ & $(20,10)$ & $(10,30)$ & $(10,20)$ & $(10,10)$ \\
\hline 1.6 & 0.00160 & 0.00188 & -0.00469 & -0.00303 & 0.00715 & -0.00410 & -0.00496 & -0.02129 & 0.00736 \\
& $(0.00344)$ & $(0.00352)$ & $(0.00538)$ & $(0.00386)$ & $(0.00517)$ & $(0.00468)$ & $(0.00942)$ & $(0.08441)$ & $(0.01113)$ \\
\hline 1.7 & -0.00195 & 0.00293 & 0.00646 & -0.00757 & -0.00113 & -0.00419 & 0.00658 & -0.01512 & 0.01178 \\
& $(0.00263)$ & $(0.00377)$ & $(0.00733)$ & $(0.00332)$ & $(0.00414)$ & $(0.00516)$ & $(0.00659)$ & $(0.05739)$ & $(0.00888)$ \\
\hline 1.8 & -0.00652 & 0.00396 & 0.01069 & -0.00538 & 0.01011 & 0.01939 & 0.01411 & 0.00935 & -0.01832 \\
& $(0.01033)$ & $(0.00405)$ & $(0.00631)$ & $(0.00307)$ & $(0.00508)$ & $(0.00712)$ & $(0.00673)$ & $(0.05318)$ & $(0.00788)$ \\
\hline 1.9 & 0.00395 & -0.00454 & -0.00186 & 0.00632 & 0.00683 & 0.01388 & -0.00120 & 0.00747 & -0.00133 \\
& $(0.00300)$ & $(0.00308)$ & $(0.00523)$ & $(0.00357)$ & $(0.00541)$ & $(0.00833)$ & $(0.00644)$ & $(0.00683)$ & $(0.00749)$ \\
\hline 2.1 & -0.00002 & -0.02159 & -0.00800 & 0.00874 & -0.00094 & 0.00568 & 0.00894 & 0.00107 & 0.01708 \\
& $(0.00427)$ & $(0.10328)$ & $(0.00623)$ & $(0.00452)$ & $(0.00466)$ & $(0.00664)$ & $(0.00669)$ & $(0.00513)$ & $(0.00795)$ \\
\hline 2.2 & 0.00566 & 0.00333 & 0.00021 & 0.00780 & -0.00053 & -0.02459 & 0.00300 & -0.00361 & -0.02646 \\
& $(0.00297)$ & $(0.00283)$ & $(0.00646)$ & $(0.00377)$ & $(0.00394)$ & $(0.04191)$ & $(0.00629)$ & $(0.02297)$ & $(0.06832)$ \\
\hline 2.3 & -0.00818 & 0.00560 & 0.00986 & -0.02177 & -0.00261 & 0.01025 & -0.00400 & 0.01435 & -0.00508 \\
& $(0.00579)$ & $(0.00273)$ & $(0.00670)$ & $(0.04988)$ & $(0.00402)$ & $(0.00720)$ & $(0.01227)$ & $(0.00781)$ & $(0.00990)$ \\
\hline 2.4 & -0.00104 & -0.00325 & -0.01584 & 0.00427 & -0.03511 & -0.02792 & 0.01112 & -0.00714 & -0.03669 \\
& $(0.00338)$ & $(0.00358)$ & $(0.02352)$ & $(0.00506)$ & $(0.12531)$ & $(0.02757)$ & $(0.00656)$ & $(0.05474)$ & $(0.11735)$ \\
\hline 2.5 & -0.02042 & 0.00051 & -0.05331 & 0.00488 & -0.02950 & -0.00573 & 0.00688 & -0.00505 & 0.01175 \\
& $(0.03452)$ & $(0.00329)$ & $(0.11499)$ & $(0.00371)$ & $(0.04784)$ & $(0.00599)$ & $(0.00477)$ & $(0.00516)$ & $(0.00928)$ \\
\hline 3 & -0.04401 & -0.02920 & -0.04264 & -0.03270 & -0.01497 & -0.05624 & -0.06668 & 0.05340 & -0.02963 \\
& $(0.02940)$ & $(0.01602)$ & $(0.11258)$ & $(0.04416)$ & $(0.02869)$ & $(0.13216)$ & $(0.18385)$ & $(0.06810)$ & $(0.04569)$ \\
\hline 4 & -0.08250 & -0.02272 & -0.01788 & -0.01816 & -0.03934 & -0.05107 & 0.02253 & -0.01600 & -0.04071 \\
& $(0.03120)$ & $(0.01323)$ & $(0.04550)$ & $(0.05063)$ & $(0.13930)$ & $(0.09691)$ & $(0.11411)$ & $(0.07030)$ & $(0.10216)$ \\
\hline
\end{tabular}

Table1: Biases and (MSE)s of the MLEs of $R$, for $a=2, b=2$, and different values of $c$

\begin{tabular}{|c|c|c|c|c|c|c|c|c|c|}
\hline $\begin{array}{c}(\mathrm{m}, \mathrm{n}) \rightarrow \\
\mathrm{c} \downarrow\end{array}$ & $(30,30)$ & $(30,20)$ & $(30,10)$ & $(20,30)$ & $(20,20)$ & $(20,10)$ & $(10,30)$ & $(10,20)$ & $(10,10)$ \\
\hline 1.6 & -0.00894 & -0.00051 & -0.02141 & 0.02561 & 0.00093 & -0.01142 & 0.02449 & -0.00806 & -0.00067 \\
& $(0.00536)$ & $(0.00505)$ & $(0.00862)$ & $(0.06577)$ & $(0.00648)$ & $(0.00968)$ & $(0.01180)$ & $(0.00994)$ & $(0.01311)$ \\
\hline 1.7 & 0.00223 & 0.01084 & -0.01395 & 0.02439 & 0.01601 & 0.00518 & 0.00762 & 0.02317 & -0.00405 \\
& $(0.00428)$ & $(0.03039)$ & $(0.00794)$ & $(0.04779)$ & $(0.01122)$ & $(0.02929)$ & $(0.00689)$ & $(0.02399)$ & $(0.01476)$ \\
\hline 1.8 & 0.00525 & 0.00700 & 0.00998 & -0.03445 & 0.00316 & 0.02482 & 0.01503 & 0.00841 & 0.02735 \\
& $(0.00383)$ & $(0.00484)$ & $(0.00881)$ & $(0.00628)$ & $(0.00600)$ & $(0.10408)$ & $(0.00947)$ & $(0.01061)$ & $(0.01075)$ \\
\hline 1.9 & 0.00318 & 0.01384 & -0.01125 & 0.00115 & 0.00500 & 0.00859 & 0.00155 & 0.00756 & 0.04176 \\
& $(0.00422)$ & $(0.00651)$ & $(0.00699)$ & $(0.00511)$ & $(0.00574)$ & $(0.00863)$ & $(0.00855)$ & $(0.00968)$ & $(0.19672)$ \\
\hline 2.1 & -0.00071 & -0.00698 & -0.00825 & 0.00895 & -0.00316 & -0.01272 & 0.02543 & 0.00068 & -0.00387 \\
& $(0.00496)$ & $(0.00519)$ & $(0.01161)$ & $(0.00138)$ & $(0.00630)$ & $(0.01099)$ & $(0.00993)$ & $(0.01023)$ & $(0.01605)$ \\
\hline 2.2 & 0.00174 & 0.01184 & -0.00745 & 0.01298 & 0.01729 & 0.03452 & 0.02671 & 0.02793 & 0.00335 \\
& $(0.00601)$ & $(0.04628)$ & $(0.00675)$ & $(0.01710)$ & $(0.02688)$ & $(0.10519)$ & $(0.01055)$ & $(0.04221)$ & $(0.01348)$ \\
\hline 2.3 & 0.04997 & -0.01035 & 0.00490 & 0.02085 & 0.01264 & 0.00419 & 0.00366 & 0.01446 & 0.00144 \\
& $(0.07521)$ & $(0.01416)$ & $(0.00883)$ & $(0.00340)$ & $(0.00601)$ & $(0.02833)$ & $(0.00843)$ & $(0.01465)$ & $(0.01268)$ \\
\hline 2.4 & 0.00578 & 0.01086 & 0.00681 & 0.00961 & 0.04411 & -0.00759 & 0.02362 & 0.01448 & -0.00517 \\
& $(0.03098)$ & $(0.03569)$ & $(0.02232)$ & $(0.00912)$ & $(0.07843)$ & $(0.00856)$ & $(0.08939)$ & $(0.01167)$ & $(0.01309)$ \\
\hline 2.5 & -0.00903 & 0.05967 & -0.01382 & 0.00244 & 0.03839 & 0.00148 & 0.01188 & 0.01131 & -0.00679 \\
& $(0.00507)$ & $(0.12854)$ & $(0.00842)$ & $(0.00552)$ & $(0.12965)$ & $(0.03331)$ & $(0.01048)$ & $(0.01996)$ & $(0.01214)$ \\
\hline 3 & -0.01090 & 0.06576 & 0.03458 & 0.06911 & 0.03340 & 0.05936 & 0.05115 & 0.03324 & 0.01505 \\
& $(0.03660)$ & $(0.16607)$ & $(0.08306)$ & $(0.00613)$ & $(0.01719)$ & $(0.14885)$ & $(0.06400)$ & $(0.06344)$ & $(0.04383)$ \\
\hline 4 & 0.02155 & 0.07522 & 0.03231 & 0.03820 & 0.04210 & -0.01316 & 0.02908 & 0.02143 & 0.01231 \\
& $(0.03128)$ & $(0.22516)$ & $(0.22089)$ & $(0.04950)$ & $(0.05290)$ & $(0.02619)$ & $(0.12232)$ & $(0.03249)$ & $(0.14150)$ \\
\hline
\end{tabular}

\section{References}

[1] Deiri, E., (2011). Estimation of $P(Y<X)$ for Exponential Distribution in the Presence of Two Outliers. International Journal of Academic Research, 3, 508-514.

[2] Deiri, E., (2011), Estimation of $P(Y<X)$ for Generalized Exponential Distribution in the Presence of Two Outliers when Scale Parameters is Known. International Journal of Academic Research, 3, 1179-1185.

[3] Deiri, E., (2011), Estimation of Parameters of the Gamma Distribution in the Presence of Two Outliers. International Journal of Academic Research, 3, 846-852.

[4] Dixit, U.J., (1989), Estimation of Parameters of the Gamma Distribution in the Presence of Outliers. Communications in Statistics - Theory and Methods, 18, 3071-3085.

[5] Dixit, U.J., Moor, K.L.and Barnett, V. (1996), On the Estimation of the Power of the Scale Parameter of the Exponential Distribution in the Presence of Outlier Generated from Uniform Distribution. Metron, 54, 201-211.

[6] Dixit, U.J.and Nasiri, P.F. (2001), Estimation of Parameters of the Exponential Distribution with Presence of Outliers Generated from Uniform Distribution. Metron, 49(3-4), 187-198.

[7] Jabbari Khamnei, H., Abolhasani, A. and Fathipour, P. (2012), Reliability for Six Parameter Generalized Burr XII Distribution with Transformation Method, Accepted in the Journal of Advances and Applications in Statistics.

[8] Nasiri,P.F.and Pazira, H. (2010), Bayesian and Non-Bayesian Estimations on the Generalized Exponential Distribution in the Presence of Outliers, Journal of Statistical Theory and Practice, $4(3), 453-475$. 